\title{
Free energy of solid solutions and phase diagrams via quasiharmonic lattice dynamics
}

\author{
N. L. Allan, ${ }^{1}$ G. D. Barrera, ${ }^{2}$ R. M. Fracchia, ${ }^{3}$ M. Yu. Lavrentiev,,${ }^{1} *$ M. B. Taylor, ${ }^{1}$ I. T. Todorov, ${ }^{1}$ and J. A. Purton ${ }^{4}$ \\ ${ }^{1}$ School of Chemistry, University of Bristol, Cantock's Close, Bristol BS8 1TS, United Kingdom \\ ${ }^{2}$ Departamento de Química, Universidad Nacional de la Patagonia SJB, Ciudad Universitaria, \\ (9000) Comodoro Rivadavia, Argentina \\ ${ }^{3}$ Universidad de Buenos Aires, Facultad de Ciencias Exactas y Naturales, Departamento de Química Inorgánica, \\ Analítica y Química Física, Pabellón 2, Ciudad Universitaria, 1428 Buenos Aires, Argentina \\ ${ }^{4}$ CLRC, Daresbury Laboratory, Warrington, Cheshire WA4 4AD, United Kingdom
}

(Received 31 October 2000; published 5 February 2001)

\begin{abstract}
We show how a configurational lattice dynamics technique, in which the free energy of a number of configurations is determined directly by means of a fully dynamic structural minimization, can be used to calculate thermodynamic properties of solid solutions and phase diagrams. No assumptions are made as to the nature of the solution and both configurational and vibrational entropy contributions are determined directly. Only a small number of configurations are required. We illustrate the method using $\mathrm{MnO} / \mathrm{MgO}$, for which our results support the recent experiments of Wood, Hackler, and Dobson [Contrib. Mineral. Petrol. 115, 438 (1994)] who, in contrast to previous workers, suggest the formation of a complete solid solution at temperatures only above $1100 \mathrm{~K}$.
\end{abstract}

DOI: 10.1103/PhysRevB.63.094203

PACS number(s): 61.43.-j, 81.05.Je, 81.30.Dz

Solid solutions, alloys, and grossly nonstoichiometric compounds present considerable challenges to the theoretician, as does the calculation of absolute free energy. Ceramic solid solutions in particular are often strongly nonideal and approaches such as the cluster variation method (CVM), ${ }^{1}$ widely used for metallic alloys, often perform poorly. In addition, despite the importance of accurate thermodynamic data for oxide solid solutions in such areas as ceramic fabrication and design and mineralogy, accurate thermodynamic data are rare.

We have recently developed a highly efficient method for the fully dynamic structure optimization of large unit cells ${ }^{2}$ that uses lattice statics and quasiharmonic lattice dynamics (QLD). We calculate the full set of free-energy first derivatives analytically, and a full minimization of the free energy with respect to all structural variables for large unit cells is possible. The accurate calculation of the free energy via QLD is quick and computationally efficient and does not resort to lengthy thermodynamic integration. Here we show this can be used for the free energies of solid solutions (including $\Delta H_{\text {mix }}$ and $\Delta S_{\text {mix }}$ ) and phase diagrams. The method is readily extended to elevated $T$ and high $P$; no a priori assumptions are made regarding the configurational entropy contribution and the vibrational contribution is also evaluated straightforwardly.

We illustrate our approach using $\mathrm{MnO} / \mathrm{MgO}$, for which not only are there several sets of experimental enthalpy data ${ }^{3,4}$ but also conflicting reports of the phase diagram. As shown in Ref. 5, the experiments of Raghavan, Iyengar, and Abraham ${ }^{6}$ suggest a consolute temperature, $T_{c}$, as low as $600 \mathrm{~K}$, whereas the results of Wood, Hackler, and Dobson ${ }^{7}$ are consistent with a much larger $T_{c}(\approx 1100 \mathrm{~K})$ and a markedly asymmetric phase diagram. The data of Ref. 6 are indeed surprising given the $\mathrm{CaO} / \mathrm{MgO}$ phase diagram, in which there is a large two-phase region, and the mismatch in ionic radii between $\mathrm{Mn}^{2+}$ and $\mathrm{Mg}^{2+}$, which is substantial although smaller than between $\mathrm{Ca}^{2+}$ and $\mathrm{Mg}^{2+}$. A further aim of this paper is to attempt to resolve this issue.

In principle the solid solution can assume any state, i.e., each atom can be at any position. However, the only states of practical importance away from the melting point will be in the bottom of $K$ local minima in the energy of the system, i.e., they will correspond to a given configuration. For each configuration $k$, we suppose there are $n_{k}$ states that correspond to small or moderate changes in the internal and external lattice strains. We ignore states corresponding to large changes in these strains, which have high energies and so do not contribute significantly to the equilibrium properties of the system. We designate a state of the system by the pair $(k l)$, where $k=1 \ldots K$ labels the configuration and $l$ $=1 \ldots n_{k}$ is the detailed state within that. The energy of such a state can be denoted $\epsilon_{k l}$. The partition function $Z$ is

$$
Z=\sum_{k}^{K} \sum_{l}^{n_{k}} \exp \left(-\beta \epsilon_{k l}\right), \quad \beta=1 /\left(k_{B} T\right),
$$

and the ensemble average of an observable $A$, which has value $A_{k l}$ in state $(k l)$ is

$$
\langle A\rangle=\frac{1}{Z} \sum_{k}^{K} \sum_{l}^{n_{k}} A_{k l} \exp \left(-\beta \epsilon_{k l}\right)
$$

Defining

$$
Z_{k}=\sum_{l}^{n_{k}} \exp \left(-\beta \epsilon_{k l}\right), \quad A_{k}=\frac{1}{Z_{k}} \sum_{l}^{n_{k}} A_{k l} \exp \left(-\beta \epsilon_{k l}\right),
$$

it can be seen that $Z_{k}$ and $A_{k}$ are the partition function and ensemble average of $A$ pertaining to a particular configuration $k$, and $\langle A\rangle$ may be rewritten

$$
\langle A\rangle=\frac{\sum_{k}^{K} A_{k} Z_{k}}{\sum_{k}^{K} Z_{k}} .
$$


The appropriate thermodynamic potential for the configuration $k$ is given by

$$
F_{k}=-k_{B} T \ln Z_{k} \text {. }
$$

Substituting for $Z_{k}$ in Eqs. (1) and (4) respectively gives

$$
F=-k_{B} T \ln \sum_{k}^{K} \exp \left(-\beta F_{k}\right), \quad\langle A\rangle=\frac{\sum_{k}^{K} A_{k} \exp \left(-\beta F_{k}\right)}{\sum_{k}^{K} \exp \left(-\beta F_{k}\right)} .
$$

We thus have expressions for the averages of any thermodynamic observable $A$ and the free energy $F$ in terms of thermodynamic quantities obtained at particular configurations. The thermodynamic averaging is performed over the results of a set of full free-energy minimizations of different arrangements of the cations within a supercell. Our configurational lattice dynamics approach to solid solutions uses the isobaric-isothermal ensemble, in which the corresponding results are

$$
H=\frac{\sum_{k}^{K} H_{k} \exp \left(-\beta G_{k}\right)}{\sum_{k}^{K} \exp \left(-\beta G_{k}\right)}, \quad G=-k_{B} T \ln \sum_{k}^{K} \exp \left(-\beta G_{k}\right),
$$

where $G_{k}$ is the free energy for the relaxed structure of each possible cation arrangement. In practice we rewrite $G$ as

$$
G=-k_{B} T \ln K-k_{B} T \ln \left(\sum_{k}^{K} \exp \left(-\beta G_{k}\right) / K\right),
$$

where $K$ is the total number of possible configurations for the supercell considered. The first term in Eq. (8) thus represents the ideal term, and the second the deviation from ideality. We note that previous work $^{8,9}$ has assumed just one, often the most regular, arrangement and calculated its enthalpy, with or possibly without relaxation, either using lattice statics or by an $a b$ initio method. For other than the smallest supercells (16 atoms) it is impractical to sum over all $K$ configurations and all summations in Eqs. (7) and (8) are restricted to $K^{\prime}$ configurations chosen at random; $K$ in the second term of Eq. (8) is replaced by $K^{\prime}$. We show later convergence can be reached using a manageable value of $K^{\prime}$.

Figure 1 shows calculated values of $\Delta H_{\text {mix }}$ at $1000 \mathrm{~K}$ for $\mathrm{MnO} / \mathrm{MgO}$, using potentials from Ref. $10 . \Delta H_{\text {mix }}$ was determined using QLD and a unit cell of 128 atoms and 250 randomly chosen arrangements. The enthalpies of mixing for 64-atom cells (averaging over either 475 configurations or over 32), and 32-atom cells (using only 32 arrangements) are almost identical but substantially smaller than those from a 16-ion cell (averaging over all arrangements). ${ }^{11}$ A previous study $^{9}$ of $\mathrm{MnO} / \mathrm{MgO}$ used the same potentials with a 16-ion cell and often a single configuration, both of which are insufficient for convergence. The size of the cell is more important than the number of configurations. Nevertheless, the small number of configurations required is striking. Also shown in Fig. 1 are values of $\Delta H_{\text {mix }}$ at $1000 \mathrm{~K}$ obtained using an exchange Monte Carlo simulation ${ }^{12}$ (MCX) in which random moves of each atom are attempted as well as

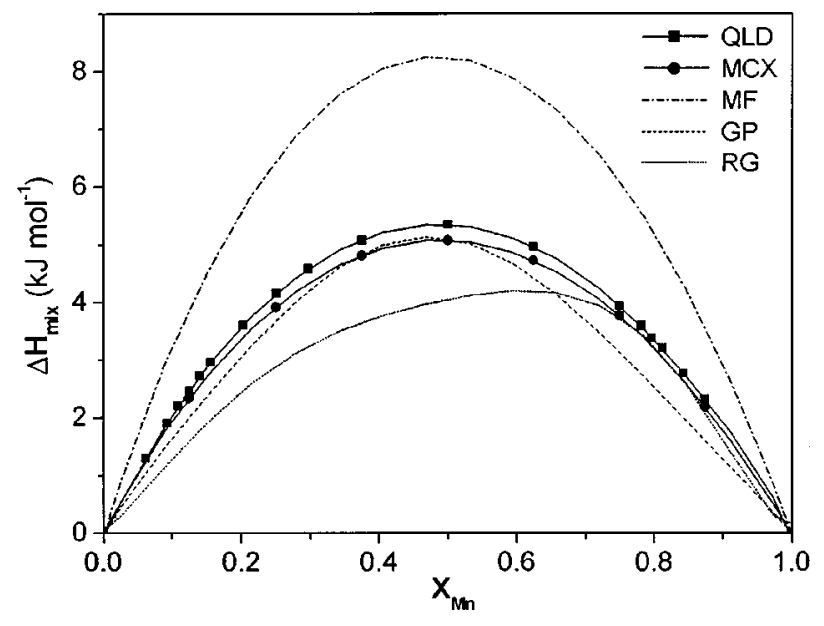

FIG. 1. Calculated values of $\Delta H_{\text {mix }}$ at $1000 \mathrm{~K}$ for $\mathrm{MnO} / \mathrm{MgO}$ using configurationally averaged lattice dynamics (QLD). For comparison purposes, values obtained using exchange Monte Carlo and the mean-field theory as described in the text, are also given. Two sets of experimental data (RG, Ref. 3, GP, Ref. 4) are shown.

random exchanges between $\mathrm{Mn}$ and $\mathrm{Mg}$ ions. These expensive calculations use a box size of 512 ions, and $4 \times 10^{7}$ steps, following an equilibration of $1 \times 10^{7}$ steps. There is excellent agreement between QLD and MCX, even though the former uses vastly less configurations than the latter and neglects higher-order anharmonic terms. QLD is a very efficient means of allowing for thermal effects. The calculated $\Delta H_{\text {mix }}$ at $1000 \mathrm{~K}$ is symmetric with a maximum $\approx 5.4$ $\mathrm{kJ} \mathrm{mol}^{-1}$. Agreement with Ref. 4 is good; the results do not show the asymmetry reported in Ref. 3 .

For comparison values calculated using a "mean-field" approach ${ }^{13}$ are also plotted in Fig. 1. If local structural relaxation or clustering is important, then these mean-field results will be poor, since these effects are averaged out in such a treatment. Figure 1 shows that this is indeed the case. The effects of the relaxation of the local atomic environments of each cation can also be seen by repeating the calculation without this relaxation. In the absence of relaxation of the basis atoms in the supercell the enthalpy of mixing at $1000 \mathrm{~K}$ for a $50 / 50$ composition is $\approx 9 \mathrm{~kJ} \mathrm{~mol}^{-1}$, again far in excess of our value of $5.4 \mathrm{~kJ} \mathrm{~mol}^{-1}$.

Our results for $\Delta H_{\text {mix }}$ are sufficiently encouraging that we have examined the use of the QLD approach for entropies of mixing via Eq. (7) and differentiation with respect to $T$. Entropies of mixing, which should be a sensitive test for our approach, are shown at $T=1000 \mathrm{~K}$ in Fig. 2(a). Figure 2(b) shows the rapid convergence of $\Delta S_{\text {mix }}$ for a 50/50 composition with cell size and with number of configurations. Use of just one configuration $\left(K^{\prime}=1\right)$ leads to a very poor estimate, ${ }^{14}$ as can be seen from Fig. 2(b). For each cell size, results for two different sets of randomly chosen configurations are shown. The QLD approach thus produces adequate convergence even for $\Delta S_{\text {mix }}$ with relatively small cells. In this case the total entropy of mixing is close to the ideal entropy of mixing.

It is important to stress that $\Delta S_{\text {mix }}$ includes both configurational and vibrational contributions; for dilute solid solu- 

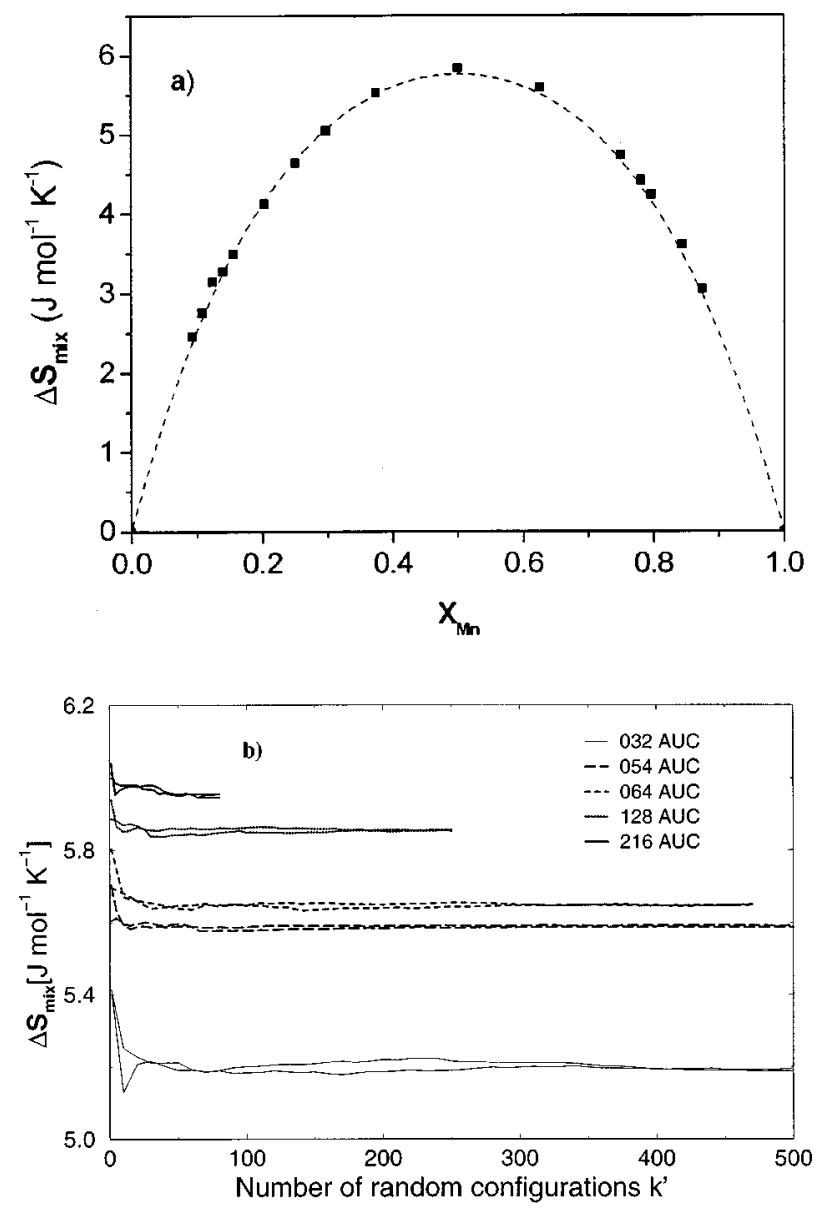

FIG. 2. (a) Calculated values of $\Delta S_{\text {mix }}$ at $1000 \mathrm{~K}$ as a function of composition. The ideal values are also shown. (b) Calculated values of $\Delta S_{\text {mix }}$ at $1000 \mathrm{~K}$ for a $50 / 50$ mixture as a function of cell size and number of configurations. For each cell size, results for two different sets of randomly chosen configurations are shown.

tions the relative contributions of each can be estimated from the calculated point-defect entropies at constant pressure, $s_{p}$, of a $\mathrm{Mn}^{2+}$ substitutional defect in $\mathrm{MgO}$ or a $\mathrm{Mg}^{2+}$ defect in $\mathrm{MnO}$, calculated using a large supercell (here 216 ions). ${ }^{15}$ When a $\mathrm{Mg}^{2+}$ ion in $\mathrm{MgO}$ is replaced by $\mathrm{Mn}^{2+}, s_{p}$ is $\approx 18.4$ $\mathrm{J} \mathrm{K}^{-1} \mathrm{~mol}^{-1}$ at $1000 \mathrm{~K}$. This vibrational contribution is positive since it is dominated by the heavier $\mathrm{Mn}^{2+}$ and the expansion of the lattice it produces, both of which decrease frequencies. Overall, $\Delta S_{\text {mix }}$ is larger than the "ideal" value and, using $s_{p}$, we estimate a vibrational contribution to $\Delta S_{\text {mix }}$ of $0.14 \mathrm{~J} \mathrm{~mol}^{-1} \mathrm{~K}^{-1}$ for a composition $12.5 \% \mathrm{MnO}$, which is $\approx 4.5 \%$ of the total value. When a $\mathrm{Mn}^{2+}$ ion in $\mathrm{MnO}$ is replaced by $\mathrm{Mg}^{2+}, s_{p} \approx-14.8 \mathrm{~J} \mathrm{~mol}^{-1} \mathrm{~K}^{-1}$ at $1000 \mathrm{~K}$, and for a composition $12.5 \% \mathrm{MgO}$, the vibrational contribution to $\Delta S_{\text {mix }}$ is $\approx 7 \%$ of the total value. The vibrational entropies for the lowest energy configurations found for the 50/50 mixture suggest a vibrational contribution to $\Delta S_{\text {mix }}$ also of $\approx 7 \%$, of the same order as for the two dilute compositions. As often assumed, $\Delta H_{\text {mix }}$ and $\Delta S_{\text {mix }}$ vary only slightly with $T$.

The calculation of the free energy of mixing presents a severe test of our model since $\Delta H_{\text {mix }}$ and $-T \Delta S_{\text {mix }}$ are often very close in magnitude. Larger cell sizes and a greater num-
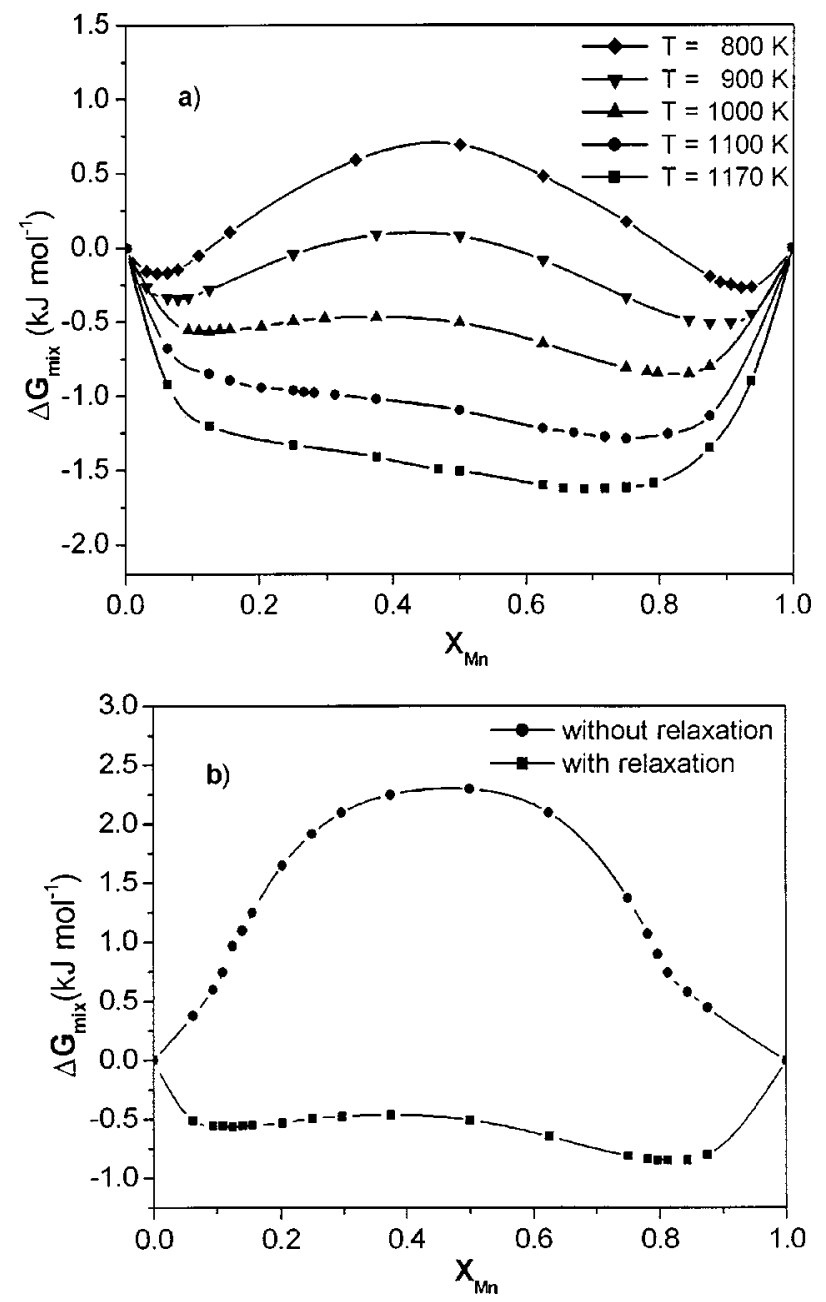

FIG. 3. Calculated values of $\Delta G_{\text {mix }}$. Results for a 128-atom cell (a) as a function of composition over a wide range of $T$ and (b) at $1000 \mathrm{~K}$ as a function of composition, with and without atomic relaxation.

ber of configurations are required. For $\mathrm{MnO} / \mathrm{MgO}$ we have found that a cell size of 128 atoms and 250 configurations are sufficient to ensure adequate convergence in the positions of the two minima in the $\Delta G_{\text {mix }}$ vs composition curve. Figure 3(a) shows the calculated variation of $\Delta G_{\text {mix }}$ for this cell size over a wide range of $T$. The crucial importance of allowing for atomic relaxation is shown strikingly in Fig. 3(b), which compares calculated $\Delta G_{\text {mix }}$ vs composition curves at $1000 \mathrm{~K}$ with and without relaxation. In the absence of relaxation $\Delta G_{\text {mix }}$ is positive for all compositions studied.

From Fig. 3(a) and the construction of common tangents to the curves with a double minimum we extract the calculated phase diagram shown in Fig. 4. We estimate the consolute temperature to lie between 1160 and $1170 \mathrm{~K}$, and thus our results provide strong support for the experimental results of Wood, Hackler, and Dobson, ${ }^{7}$ ruling out the formation of a complete solid solution at temperatures as low as $600 \mathrm{~K}$, as suggested by the data of Raghavan, Iyengar, and Abraham. ${ }^{6}$ Although our calculated $\Delta H_{\text {mix }}$ vs composition curve is symmetric, our calculated phase diagram possesses a marked asymmetry with $\mathrm{MnO}$ less soluble in $\mathrm{MgO}$ than 


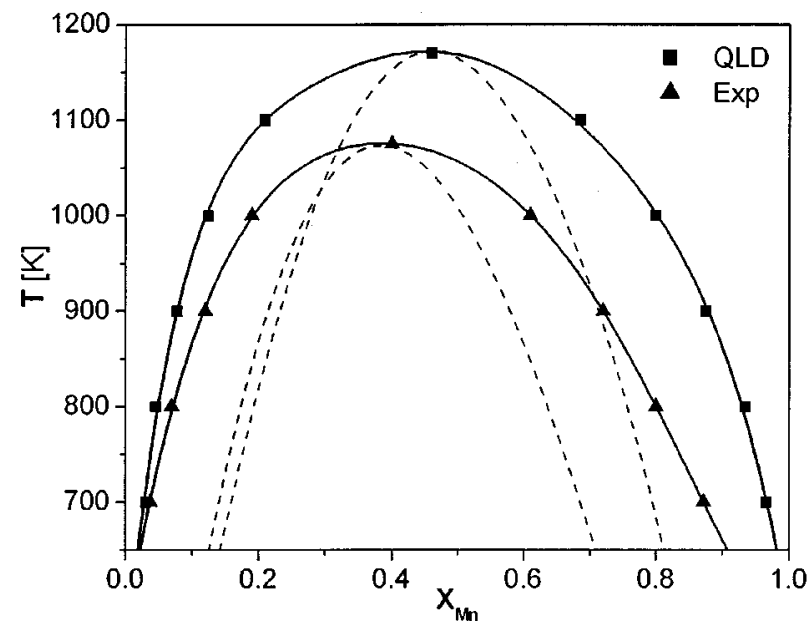

FIG. 4. Calculated phase diagram and spinodal for $\mathrm{MnO} / \mathrm{MgO}$.

$\mathrm{MgO}$ in $\mathrm{MnO}$. The same type of asymmetry is also observed in the $\mathrm{CaO} / \mathrm{MgO}$ system, with the smaller cation more soluble in $\mathrm{CaO}$ than the larger cation in $\mathrm{MgO}$. The phase diagram obtained from the measurements of Wood, Hackler, and Dobson ${ }^{7}$ is more asymmetric than that calculated. It is worth noting the conclusions of de Villiers, Buseck, and Steyn, ${ }^{5}$ who emphasize the large experimental errors and suggest that the asymmetry should be smaller.

A common model for solid solutions assumes a subregular solution and the Margules approximation. ${ }^{16} \mathrm{~A}$ much better fit to our results for $G_{\text {excess }}$ is obtained including also a fourth-order term. It is straightforward to extract the spinodal, which defines the region where a single phase is kinetically unstable with respect to two separate phases. The cal- culated spinodal is given in Fig. 4 together with that extracted by de Villiers, Buseck, and Steyn ${ }^{5}$ from the experimental data of Ref. 7.

In summary, we have demonstrated how the rapid calculation of the free energy via quasiharmonic lattice dynamics can be used to calculate thermodynamic properties of solutions over wide ranges of $T$ including $\Delta H_{\text {mix }}$ and $\Delta S_{\text {mix }}$, and phase diagrams. Disorder problems are often tackled by using a general Ising model, simplified by limiting interactions to a short range and a finite number of multisite couplings. Such an approach is awkward to parametrize for ionic solids, where relaxation is crucial, and to apply over a range of $T$ and $P$. It is not readily generalized to less symmetric structures, to which we also wish to apply the general methodology outlined here. In our methodology, no assumptions are made as to the nature of the solid solution. Calculated entropies of mixing include both configurational and vibrational contributions. It is an attractive strategy below the Debye temperature $\left(\Theta_{D}\right)$ where classical Monte Carlo and molecular dynamics fail due to neglect of quantum effects and so is particularly appropriate here where $\Theta_{D}$ for $\mathrm{MgO}$ is $900 \mathrm{~K}$ and for $\mathrm{MnO}$ is $500 \mathrm{~K}$. The technique is limited only by the accuracy of the quasiharmonic approximation, which breaks down with increasing amplitude of vibration and hence at high $T$; it breaks down for oxides typically around two-thirds of the melting point. Further work is in progress to develop this technique, which we denote configurational lattice dynamics, and apply it to more complex systems.

This work was funded by EPSRC Grants No. GR/ M34799, GR/M53899, and ANPCyT Grant BID No. 802/ OC-AR-PICT0361. G.D.B. acknowledges support from CONICET and the Fundación Antorchas. We would like to thank Hugh Barron for useful discussions.
*On leave from Institute of Inorganic Chemistry, 630090 Novosibirsk, Russia.

${ }^{1}$ D. de Fontaine, Solid State Phys. 47, 33 (1994).

${ }^{2}$ M. B. Taylor, G. D. Barrera, N. L. Allan, and T. H. K. Barron, Phys. Rev. B 56, 14380 (1997).

${ }^{3}$ S. Raghavan, Ph.D. thesis, Indian Institute of Science, Bangalore, India, 1977.

${ }^{4}$ H. Gripenberg, S. Seetharaman, and L.-I. Staffansson, Chem. Scr. 13, $162(1978-79)$.

${ }^{5}$ J. P. R. de Villiers, P. R. Buseck, and H. S. Steyn, Miner. Mag. 62, 333 (1998).

${ }^{6}$ S. Raghavan, G. N. K. Iyengar, and K. P. Abraham, J. Chem. Thermodyn. 17, 585 (1985).

${ }^{7}$ B. J. Wood, R. T. Hackler, and D. P. Dobson, Contrib. Mineral. Petrol. 115, 438 (1994).

${ }^{8}$ K. D. Heath, W. C. Mackrodt, V. R. Saunders, and M. Causà, J. Mater. Chem. 4, 825 (1994).

${ }^{9}$ M. Königstein, F. Corà, and C. R. A. Catlow, J. Solid State
Chem. 137, 261 (1998).

${ }^{10}$ G. V. Lewis and C. R. A. Catlow, J. Phys. C: Solid State Phys. 18, 1149 (1985).

${ }^{11}$ J. A. Purton, J. D. Blundy, M. B. Taylor, G. D. Barrera, and N. L. Allan, Chem. Commun. (Cambridge) 1998, 627.

${ }^{12}$ For our implementation of exchange Monte Carlo see N. L. Allan, G. D. Barrera, M. Yu. Lavrentiev, I. T. Todorov, and J. A. Purton, J. Mater. Chem. 11, 63 (2001).

${ }^{13}$ A "hybrid" ion is introduced, for which the non-Coulombic potentials are a linear combination of the potentials for $\mathrm{Mn}^{2+}$ and $\mathrm{Mg}^{2+}$, weighted by the site occupancies.

${ }^{14}$ For a unit cell of 128 atoms the use of one configuration may result in values of $\Delta H_{\text {mix }}, \Delta G_{\text {mix }}$, and $\Delta S_{\text {mix }}^{\text {excess }}$ as far as $10 \%$, $76 \%$, and $53 \%$, respectively, from their final values.

${ }^{15}$ M. B. Taylor, G. D. Barrera, N. L. Allan, T. H. K. Barron, and W. C. Mackrodt, Faraday Discuss. 106, 377 (1997).

${ }^{16}$ E.g., J. B. Thompson, Researches in Geochemistry, edited by P. H. Abelson (Wiley, New York, 1967), Vol. II, p. 340. 